\title{
Antiferromagnetic single-layer spin-orbit torque oscillators
}

\author{
Roberto E. Troncoso, ${ }^{1,{ }^{*}}$ Karsten Rode,${ }^{2}$ Plamen Stamenov, ${ }^{2}$ J. Michael D. Coey, ${ }^{2}$ and Arne Brataas \\ ${ }^{1}$ Center for Quantum Spintronics, Department of Physics, Norwegian University of Science and Technology, NO-7491 Trondheim, Norway \\ ${ }^{2}$ CRANN, AMBER and School of Physics, Trinity College Dublin, Dublin 2, Ireland
}

(Received 31 August 2018; revised manuscript received 13 February 2019; published 27 February 2019)

\begin{abstract}
We show how a charge current through a single antiferromagnetic layer can excite and control self-oscillations. Sustained oscillations with tunable amplitudes and frequencies are possible in a variety of geometries using certain classes of noncentrosymmetric materials that exhibit finite dissipative spin-orbit torque. We compute the steady-state phase diagram as a function of the current and spin-orbit torque magnitude. The anisotropic magnetoresistance causes the conversion of the resulting AF oscillations to a terahertz AC output voltage. These findings provide an attractive and novel route to design terahertz antiferromagnetic spin-orbit torque oscillators in simple single-layer structures.
\end{abstract}

DOI: 10.1103/PhysRevB.99.054433

\section{INTRODUCTION}

Modern technologies cannot easily generate and detect radiation in the spectral range between radio waves and infrared light [1]. Typically, microwaves reach at most $100 \mathrm{GHz}$ while optical techniques cover frequencies higher than $30 \mathrm{THz}$ [2]. Despite enormous efforts [1], in this 'Terahertz gap,' efficient, cheap, chip-sized, and practical technologies do not exist [2].

Spin dynamics of antiferromagnets (AFs) have the potential to fill the terahertz gap $[3,4]$ as their spin excitation frequency lie in the required range [1]. They are robust against external fields since there is no net magnetic moment [4], but on the other hand, this implies there is no simple way to control them by magnetic fields [5]. As a result, currentinduced and -controlled AF spin dynamics has emerged [4-6]. To demonstrate their potential as enabling components in terahertz devices, we must establish how to control ultrafast switching and oscillations [7-10].

Single-layer spin-orbit torques offer such possibilities [11-14]. The origin of these torques is the relativistic spinorbit interaction. In noncentrosymmetric AFs, where the magnetic sublattices form inversion partners, a current induces a spin polarization with an alternating sign between the inversion partner lattice sites. There are recent observations of fieldlike (reactive) SOTs, dubbed Néel spin-orbit torque (NSOT), in CuMnAs [15] and $\mathrm{Mn}_{2} \mathrm{Au}$ [16]. The functional form of SOTs depends on the crystallographic symmetries [11], and we expect interesting phenomenology in a wide variety of AFs.

In addition to reactive SOTs, the transfer of angular momentum from currents to magnetization can compensate or enhance the magnetic (Gilbert) damping via dissipative ('dampinglike') torques. This can result in steady-state magnetic oscillations, also known as self-oscillations [17]. Spintransfer torques have a large dissipative component and can therefore sustain stable, high-frequency oscillations [18-21]

*r.troncoso@ntnu.no but generally require an external source of spin currents. Radically different, single-layer AF-based SOT oscillators would not need a spin polarizer and have the potential of combining stable oscillations with high speed dynamics, useful for emitters and receivers of $\mathrm{THz}$ radiation.

In this paper, we propose a route to realize currentcontrolled SOT oscillators in AFs. First, we determine the form of the SOTs based on symmetry arguments through a phenomenological approach. Second, the behavior of selfoscillations is studied for different ratios of the reactive and dissipative torques as well as its dependence on the charge current density.

This work is structured as follows. In Sec. II, we introduce the phenomenological theory to compute SOTs in AFs. In Sec. III, the antiferromagnetic dynamics is presented and is followed by Sec. IV where self-oscillations are calculated. Finally, we end with conclusions and discussions in Sec. V.

\section{SPIN-ORBIT TORQUES}

We consider a two-sublattice, noncentrosymmetric AF described by unit vectors along the magnetic moments, $\boldsymbol{m}_{a}$ and $\boldsymbol{m}_{b}$. A general form of the current-induced torque $[11,14]$ in the local approximation is $\boldsymbol{\tau}_{\alpha}=\boldsymbol{m}_{\alpha} \times \mathbf{H}_{\alpha}\left[\boldsymbol{m}_{a}, \boldsymbol{m}_{b}, \mathbf{j}\right]$, where $\alpha$ labels the sublattices. $\mathbf{H}_{\alpha}$ is the effective field that depends on the magnetization direction and is induced by the outof-equilibrium current density $\mathbf{j}$ [11]. In linear response, the torque is

$$
\boldsymbol{\tau}_{\alpha}=\boldsymbol{m}_{\alpha} \times \boldsymbol{\eta}_{\alpha}\left[\boldsymbol{m}_{a}, \boldsymbol{m}_{b}\right] \mathbf{j},
$$

where the local second-rank tensor $\boldsymbol{\eta}_{\alpha} \equiv\left(\partial \mathbf{H}_{\alpha} / \partial \mathbf{j}\right)_{\mathbf{j}=0}$ encodes all forms of current-induced torques governed by the symmetries of $\boldsymbol{\eta}_{\alpha}$. Invoking Neumann's principle [22], symmetries possessed by the point group of the crystal will be inherited by any physical quantity, we compute SOTs. In AFs, we consider symmetries that leave the magnetic sublattice site invariant $[13,23]$. Denoting $\mathcal{R}$ a symmetry rotation, the magnetization and current density obey $\boldsymbol{m}_{\alpha}^{\prime}=|\mathcal{R}| \mathcal{R} \boldsymbol{m}_{\alpha}$ and $\mathbf{j}^{\prime}=\mathcal{R} \mathbf{j}$, respectively, in the transformed frame. The 


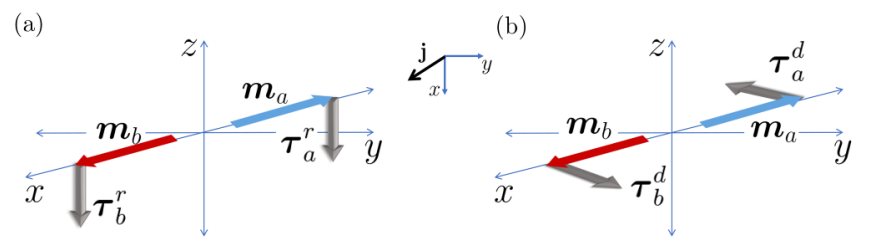

FIG. 1. The action of (a) reactive $\boldsymbol{\tau}_{a, b}^{r}$ and (b) dissipative $\boldsymbol{\tau}_{a, b}^{d}$ spin-orbit torques on the magnetic moments at bipartite lattices $\boldsymbol{m}_{a}$ and $\boldsymbol{m}_{b}$. The torques are driven by an in-plane current density $\boldsymbol{j}$.

transformation rule for the torque is $\boldsymbol{\tau}_{\alpha}^{\prime}=|\mathcal{R}| \mathcal{R} \boldsymbol{\tau}_{\alpha}$. It follows from Neumann's principle that $\boldsymbol{\eta}_{\alpha}$ must satisfy, $\boldsymbol{\eta}_{\alpha}\left[\boldsymbol{m}_{a}^{\prime}, \boldsymbol{m}_{b}^{\prime}\right]=$ $|\mathcal{R}| \mathcal{R} \eta_{\alpha}\left[\boldsymbol{m}_{a}, \boldsymbol{m}_{b}\right] \mathcal{R}^{T}$. We consider the magnetic sublattices form inversion partners within each unit cell. This assumption leads to the additional condition $\boldsymbol{\eta}_{b}\left[\boldsymbol{m}_{b}, \boldsymbol{m}_{a}\right]=-\boldsymbol{\eta}_{a}\left[\boldsymbol{m}_{a}, \boldsymbol{m}_{b}\right]$.

To proceed, we simplify the tensor $\boldsymbol{\eta}_{\alpha}$ by expanding it up to leading order in $\boldsymbol{m}_{\alpha}$, i.e., $\boldsymbol{\eta}_{\alpha}=\boldsymbol{\Lambda}_{\alpha}+\boldsymbol{\Lambda}_{\alpha}^{a} \boldsymbol{m}_{a}+\boldsymbol{\Lambda}_{\alpha}^{b} \boldsymbol{m}_{b}$ $[11,22]$. The reactive and dissipative SOTs are captured by the second- and third-rank tensors $\boldsymbol{\Lambda}_{\alpha}, \boldsymbol{\Lambda}_{\alpha}^{a}$, and $\boldsymbol{\Lambda}_{\alpha}^{b}$, respectively. The derived symmetry relations for $\boldsymbol{\eta}_{\alpha}$ imply that these tensors obey

$$
\begin{gathered}
{\left[\boldsymbol{\Lambda}_{a, b}\right]_{i j}=-|\mathcal{R}| \mathcal{R}_{i i^{\prime}} \mathcal{R}_{j j^{\prime}}\left[\boldsymbol{\Lambda}_{b, a}\right]_{i^{\prime} j^{\prime}},} \\
{\left[\boldsymbol{\Lambda}_{a, b}^{a}\right]_{i j k}=-\mathcal{R}_{i i^{\prime}} \mathcal{R}_{j j^{\prime}} \mathcal{R}_{k k^{\prime}}\left[\boldsymbol{\Lambda}_{b, a}^{b}\right]_{i^{\prime} j^{\prime} k^{\prime}},}
\end{gathered}
$$

for a specific symmetry group.

We now focus on three groups of tetragonal noncentrosymmetric materials. First, we consider systems described by a linear in momentum Dresselhaus model [13,24]. These are found in certain Heusler alloys, like the fully-compensated half-metallic ferrimagnet $\mathrm{Mn}_{2} \mathrm{Ru}_{x} \mathrm{Ga}$ (MRG) [25-27]. The large magnetocrystalline anisotropy and the lack of local inversion symmetry existing in MRG [25] imply a considerable SOT. To compute the torques we consider that symmetries belongs to the tetragonal point group $\overline{4} 2 m[23,25]$. The simplest expression for SOTs is obtained when MRG is close to the cubic phase. As detailed in Appendix A, we find that the torques on each sublattice are

$$
\begin{aligned}
\boldsymbol{\tau}_{a} & =\Lambda \boldsymbol{m}_{a} \times \boldsymbol{j}+\mathcal{T}\left\{\boldsymbol{m}_{a} \times\left[\left(\Lambda^{n} \boldsymbol{m}_{a}+\Lambda^{m} \boldsymbol{m}_{b}\right) \times \boldsymbol{j}\right]\right\}, \\
\boldsymbol{\tau}_{b} & =-\Lambda \boldsymbol{m}_{b} \times \boldsymbol{j}-\mathcal{T}\left\{\boldsymbol{m}_{b} \times\left[\left(\Lambda^{m} \boldsymbol{m}_{a}+\Lambda^{n} \boldsymbol{m}_{b}\right) \times \boldsymbol{j}\right]\right\},
\end{aligned}
$$

for an in-plane charge current $\boldsymbol{j}=\left(j_{x},-j_{y}, 0\right)$. The operator $\mathcal{T}\{\cdot\}$ switches the sign of the $z$ component, while $x$ and $y$ components are unchanged. To derive the simplest expression for the torques we have assumed $\boldsymbol{m}_{a}$ and $\boldsymbol{m}_{b}$ in MRG form spin partners (see Appendix A 1 for details). The phenomenological coefficients $\Lambda, \Lambda^{n}$, and $\Lambda^{m}$ quantify the strength of reactive and dissipative SOTs sketched in Fig. 1.

A second example are the AFs, $\mathrm{Mn}_{2} \mathrm{Au}$ [16] and CuMnAs [15]. The symmetries of the magnetic sites belongs to the polar point group $4 \mathrm{~mm}$ [13]. The SOTs for these materials are similar to the torques in MRG when the Fermi level is close to the band gap $[12,13,28]$. In fact, the result is obtained under the transformation $\mathbf{j} \rightarrow \boldsymbol{z} \times \mathbf{j}$, with $\mathbf{j}=\left(j_{x}, j_{y}, 0\right)$, and $\mathcal{T} \rightarrow I$, with $I$ the identity. Lastly, the third group of materials that we consider are part of enantiomorphic crystals [13,23]. As an example we choose systems with point group 422 . The
SOTs are found from the torques in MRG under the change $\boldsymbol{j} \rightarrow \mathbf{j}$ and $\mathcal{T} \rightarrow I$. Note that in the previous two examples we also used that the material is approximately cubic. The expression found for these groups also recovers the results given in Ref. [13]. Details and the general result for the SOTs can be found in Appendices A 1-A 3.

\section{ANTIFERROMAGNETIC DYNAMICS}

The magnetocrystalline anisotropy has two dominant components-a uniaxial anisotropy perpendicular to the AF layer and an in-plane fourfold symmetric anisotropy. Next, we introduce the Néel vector $\boldsymbol{n}=\left(\boldsymbol{m}_{a}-\boldsymbol{m}_{b}\right) / 2$ and the magnetization $\boldsymbol{m}=\left(\boldsymbol{m}_{a}+\boldsymbol{m}_{b}\right) / 2$ that satisfy $\boldsymbol{m} \cdot \boldsymbol{n}=0$ and $m^{2}+n^{2}=1$. The free magnetic energy density, including the exchange energy, is $f=-2 \omega_{\mathrm{ex}} n^{2}-\omega_{\|}^{(1)} n_{z}^{2} / 2-$ $\omega_{\perp}^{(1)}\left(n_{x}^{4}+n_{y}^{4}-6 n_{x}^{2} n_{y}^{2}\right) / 4-\omega_{\|}^{(2)} n_{z}^{4}$ in the macrospin description and employing the exchange approximation. The coupled equations of motion are

$$
\begin{gathered}
\dot{\boldsymbol{m}}=\mathbf{f}_{m} \times \boldsymbol{m}+\mathbf{f}_{n} \times \boldsymbol{n}+\alpha(\boldsymbol{m} \times \dot{\boldsymbol{m}}+\boldsymbol{n} \times \dot{\boldsymbol{n}})+\boldsymbol{\tau}_{m} \\
\dot{\boldsymbol{n}}=\mathbf{f}_{n} \times \boldsymbol{m}+\mathbf{f}_{m} \times \boldsymbol{n}+\alpha(\boldsymbol{m} \times \dot{\boldsymbol{n}}+\boldsymbol{n} \times \dot{\boldsymbol{m}})+\boldsymbol{\tau}_{n}
\end{gathered}
$$

with the thermodynamic forces $\mathbf{f}_{m} \equiv-\partial f / \partial \boldsymbol{m}$ and $\mathbf{f}_{n} \equiv$ $-\partial f / \partial \boldsymbol{n}$, and $\alpha$ the Gilbert damping constant. The SOTs exerted on the magnetization and Néel field are, respectively, defined as $\boldsymbol{\tau}_{m, n}=\left(\boldsymbol{\tau}_{a} \pm \boldsymbol{\tau}_{b}\right) / 2$. In the exchange approximation, Eq. (5) implies that the small magnetization $\boldsymbol{m}$ is a slave variable obeying $\boldsymbol{m}=\left(\dot{\boldsymbol{n}}-\boldsymbol{\tau}_{n}\right) \times \boldsymbol{n} /\left(4 \omega_{\mathrm{ex}}\right)$. Inserting the expression for $\boldsymbol{m}$ into Eq. (4), we obtain the effective equation of motion that governs the Néel field dynamics,

$$
\boldsymbol{n} \times\left[\frac{\ddot{\boldsymbol{n}}}{4 \omega_{\mathrm{ex}}}-\mathbf{f}_{n}^{\mathrm{an}}+\alpha_{\mathrm{eff}}[\boldsymbol{n}] \dot{\boldsymbol{n}}+\Lambda \boldsymbol{j}+\mathbf{X}[\boldsymbol{n}, \dot{\boldsymbol{n}}]\right]=0,
$$

with the constraint $|\boldsymbol{n}|=1$ and $\mathbf{f}_{n}^{\text {an }}$ the effective anisotropy field. We consider the simplest regime where $\Lambda^{n}$ and $\Lambda^{m}$ are comparable. $\mathbf{X}[\boldsymbol{n}, \dot{\boldsymbol{n}}]$ is a field that derives from the dissipative SOT [29] and it is characteristic of MRG. However, when the easy-plane anisotropy $\omega_{\perp}^{(1)}$ is sufficiently large, this term can be neglected from Eq. (6). The dissipative torque also modifies the Gilbert damping by leading to the effective dissipation,

$$
\alpha_{\mathrm{eff}}[\boldsymbol{n}]=\alpha-\frac{\Lambda^{n}}{2 \omega_{\mathrm{ex}}} \boldsymbol{j} \cdot \boldsymbol{n}
$$

that depends on the charge current and the Néel vector.

For a comprehensive description of the AF dynamics, we restrict our analysis to the easy-plane magnetic configuration $\left(\omega_{\|}^{(1)}<0, \omega_{\|}^{(2)} \approx 0\right.$ and $\left.\omega_{\perp}^{(1)}>0\right)$. In this case there are four in-plane degenerate states. We write $\boldsymbol{n}$ in terms of its polar $(\theta)$ and azimuthal $(\phi)$ angle. Maintaining the assumption of a dominant easy-plane anisotropy, we use the ansatz $\theta(t)=$ $\pi / 2$. Thus, Eq. (6) reduces to the scalar equation

$$
\frac{1}{4 \omega_{\mathrm{ex}}} \ddot{\phi}+\alpha_{\mathrm{eff}}[\phi] \dot{\phi}=F[\phi]
$$

that corresponds to a forced nonlinear oscillator. The current controls the effective damping $\alpha_{\text {eff }}[\phi]$ of Eq. (7) and the force 
$F[\phi]=-\partial V / \partial \phi$, where the potential

$$
V[\phi]=-\frac{\omega_{\perp}^{(1)}}{4} \cos [4 \phi]+\Lambda\left[j_{x} \cos \phi-j_{y} \sin \phi\right] .
$$

At equilibrium, when there is no current, the four degenerate ground states have azimuthal angles $\phi=-\pi, \phi=$ $-\pi / 2, \phi=0$ or $\phi=\pi / 2$.

\section{SELF-OSCILLATIONS}

We will now demonstrate the emergence of selfoscillations. This is presented for MRG, however it is extensible to the other candidate materials under simple rotations of the charge current. Furthermore, up to leading order in the amplitude of oscillations, Eq. (8) reduces to the well-studied Duffing-Van der Pol oscillator $[17,30]$. Our main result is that self-oscillations are stable in two scenarios. When the dissipative SOT $\Lambda^{n}$ is negative, self-oscillations emerge when the charge current is parallel to the $y$ or $x$ axis. In contrast, when $\Lambda^{n}>0$, the previous statement holds but stabilization occurs in a narrow window of currents. Moreover, due to the fourfold symmetry, the azimuthal angle will behave the same for currents along $x$ or $y$. Therefore, the result for self-oscillations appearing when $\boldsymbol{j} \| \hat{y}$ is replicated when $\boldsymbol{j}$ is parallel to the $x$ axis by transforming $\phi \rightarrow \phi+\pi / 2$. These results are detailed in the following.

We now consider a charge current along the $y$ axis. We first discuss the effects of the reactive torque, disregarding dynamical and hence dissipative effects. The (reactive) currentdependent force $F_{r}=\Lambda j_{y} \cos \phi$ changes the azimuthal angle where the potential $V(\phi)$ attains its minimum. For positive current densities $0 \leqslant j_{y}<j_{\mathrm{sw}} \equiv \omega_{\perp}^{(1)} / \Lambda$, there are four minima of $V(\phi)$, corresponding to the steady states as seen in Fig. 2(c). Among these, there are two degenerate configurations, $\phi_{\mathrm{ss}}^{a, b}$, which become unstable when $j_{y}>j_{\mathrm{sw}}$. The other two states are $\phi_{\mathrm{ss}}^{ \pm}$. However, when the current is sufficiently large, $j_{y} \geqslant 4 j_{\mathrm{sw}}$, the state at $\phi_{\mathrm{ss}}^{+}$is unstable and thus leaving the only stable steady state at $\phi_{\mathrm{ss}}^{-}$, see Eq. (9). For negative currents, $j_{y}<0$, the steady-state angles reverse their sign, $\phi_{\mathrm{ss}}\left(j_{y}\right)=-\phi_{\mathrm{ss}}\left(-j_{y}\right)$.

We now include the dissipative SOT and show how selfoscillations emerge from Eq. (8). The essential requirement for self-oscillations is that the dissipative spin-orbit forces compensate the magnetization dissipation. To find the onset of self-oscillations, it is sufficient to evaluate the effective damping Eq. (7) around the steady state $\phi_{\mathrm{ss}}^{-}$. The damping is then $\alpha_{\mathrm{eff}}^{y}=\alpha+\left(\Lambda^{n} / 2 \omega_{\mathrm{ex}}\right) j_{y} \sin \left[\phi_{\mathrm{ss}}\left(j_{y}\right)\right]$, which is nonlinear in $j_{y}$ because $\phi_{\mathrm{ss}}$ depends on the current. Consequently, in order to compensate the Gilbert damping, the dissipative strength has to be negative since $\phi_{\mathrm{ss}}^{-}>0$ [31]. This result is valid when the current flows in both directions $\pm \hat{y}$, since the steady-state angle is an odd function of the current. Self-oscillations set in when the current exceeds the threshold $j_{\mathrm{so}}^{(1)}=2 \omega_{\mathrm{ex}} \alpha /\left|\Lambda^{n}\right|$, where the damping satisfies $\alpha_{\mathrm{eff}}^{y}=0$.

We numerically solve Eq. (8) for the azimuthal angle $\phi(t)$ in two representative cases. In these simulations, the Gilbert damping constant is $\alpha=0.005$ and there is a small anisotropy field $\omega_{\perp}^{(1)} / \omega_{\mathrm{ex}}=10^{-2}$ consistent with the exchange approximation. The ratio of the dissipative and reactive SOTs strength is considered in the range $\left|\Lambda^{n}\right| / \Lambda \epsilon$ (a)

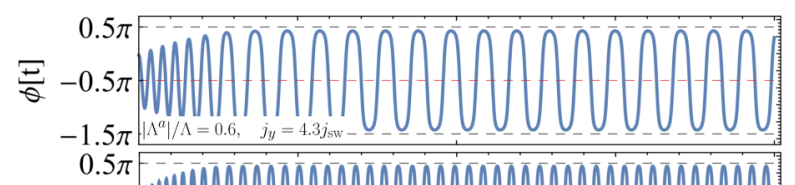

(b)

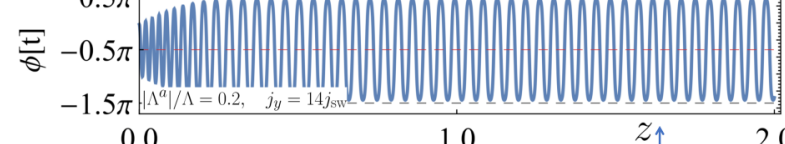

0.0

1.0

$10^{-2} \mathrm{t} / t_{0}$

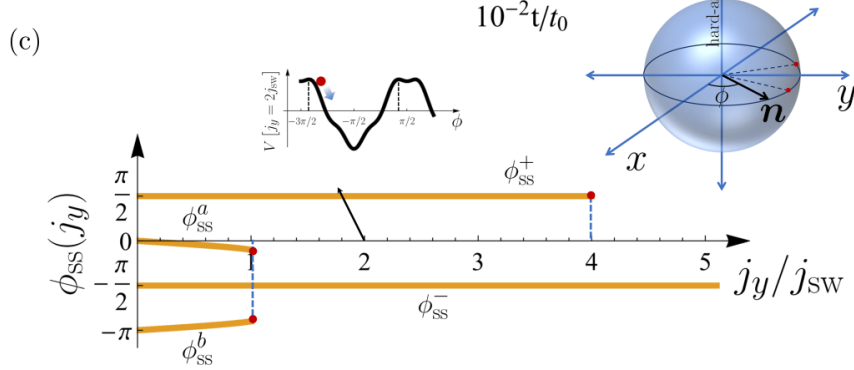

FIG. 2. Time evolution of the azimuthal angle, in units of $t_{0}=$ $1 /\left(2 \sqrt{\omega_{\mathrm{ex}} \omega_{\|}^{(1)}}\right)$, driven by a current along the $y$ direction when the Gilbert damping is $\alpha=0.005$. The dissipative SOT strength and current are (a) $\left|\Lambda^{n}\right| / \Lambda=0.6$ and $j_{y}=4.3 j_{\mathrm{sw}}$, (b) $\left|\Lambda^{n}\right| / \Lambda=0.2$ and $j_{y}=14 j_{\mathrm{sw}}$, respectively. (c) Steady-state angle as a function of current density $j_{y}$ normalized in $j_{\mathrm{sw}}$. There is a global minima at $\phi_{\mathrm{ss}}^{-}$that prevails at any current. When $0 \leqslant j_{y}<4 j_{\mathrm{sw}}$, three steady states exist (local minima) at $\phi_{\mathrm{ss}}^{a, b}$ and $\phi_{\mathrm{ss}}^{+}$, which become unstable at $j_{y}=j_{\mathrm{sw}}$ and $j_{y}=4 j_{\mathrm{sw}}$, respectively.

$[0.1,1]$. Figure 2(a) shows the time evolution of $\phi(t)$ when there is a charge current $j_{y}=4.3 j_{\mathrm{sw}}$ and $\left|\Lambda^{n}\right| / \Lambda=0.6$. As an initial condition, we slightly perturb the steady state $\phi_{\mathrm{ss}}^{-}$ so that the azimuthal angle starts at $\phi(t=0)=\phi_{\mathrm{ss}}^{-}+0.01$. Thereafter, the azimuthal angle oscillates around $\phi_{\mathrm{ss}}^{-}$with an amplitude that increases until it becomes stable. We find that the saturated amplitude is independent on the initial conditions, as long as $\phi(t=0) \neq \phi_{\mathrm{ss}}^{-}$. In a second example, displayed in Fig. 2(b), we consider the evolution of $\phi(t)$, with the same initial condition as above, but $\left|\Lambda^{n}\right| / \Lambda=0.2$ and under a larger current $j_{y}=14 j_{\mathrm{sw}}$. As we see, $\phi(t)$ stabilizes at an amplitude similar to the previous example but twice of frequency.

The numerical results can be understood intuitively. In order to prevent Néel-field oscillations from relaxing into the current-induced steady state determined by the reactive torque, a significant dissipative SOT is paramount. Realizing stable oscillations require a large charge current when the SOT is small. When $j_{y} \geqslant j_{\text {so }}^{(1)}$, and after the transient features, the terminal amplitude of the oscillations is a good order parameter to characterize the stable regimes. We compute perturbatively the equation of motion for the amplitude of oscillations $\mathcal{A}$ using multiple-scale analysis [32]. Two natural time scales dominate the evolution of $\phi(t)$, see Fig. 2(a). There are rapid precessions around $\phi_{\mathrm{ss}}^{-}$. Additionally, the amplitude of precessions evolves on a slower time scale. Averaging over the rapid precessions, to leading order in the amplitude $\mathcal{A}$, we find that the amplitude obeys the well-known GinzburgLandau equation [30],

$$
2 \dot{\mathcal{A}}=\mathcal{A}-\mathcal{A}^{3} /\left[8\left(1-j_{\mathrm{so}}^{(1)} / j_{y}\right)\right] .
$$




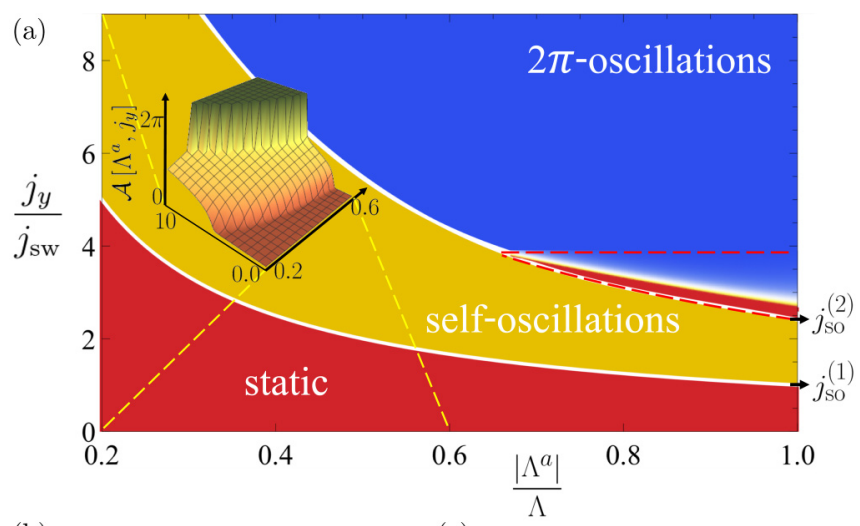

(b)

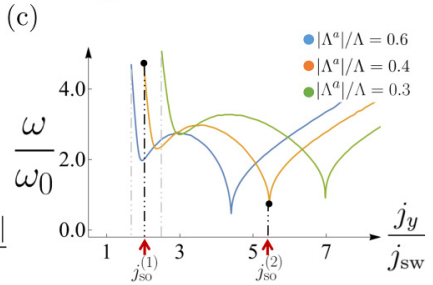

FIG. 3. (a) Terminal amplitude of self-oscillations as a function of current density $j_{y}$ and dissipative SOT strength $\left|\Lambda^{n}\right|$. (b) The threshold current $j_{\text {so }}^{(1)}$, defining the border between static and stable oscillations, for different values of $\alpha$. For $\alpha=0.005$, in (c) is shown in units of $\omega_{0}=1 / t_{0}$ the characteristic frequency of self-oscillations in terms of the current density. At the threshold $j_{\mathrm{so}}^{(1)}$, the frequencies drop to zero corresponding to the static regime. At $j_{\text {so }}^{(2)}$ the frequency reaches a finite value that change in terms of the ratio $\left|\Lambda^{n}\right| / \Lambda$. For $j_{y}>j_{\mathrm{so}}^{(2)}$, the frequency of full oscillations grows strictly with the current.

At long time scales, Eq. (10) determines the terminal amplitude $\overline{\mathcal{A}}=2 \sqrt{2} \sqrt{1-j_{\text {so }}^{(1)} / j_{y}}$. Consequently, the amplitude $\overline{\mathcal{A}} \rightarrow 0$ when $j_{y} \leqslant j_{\text {so }}^{(1)}$, reproducing the boundary between static states and self-oscillations. For large current densities $j_{y} \gg j_{\text {so }}^{(1)}$, the amplitude saturates to $\overline{\mathcal{A}} \approx 2 \sqrt{2}$. This analytical result is somewhat smaller than the value reached by the azimuthal angle in the numerical solution shown in Fig. 2. This is because an exact description of large-amplitude oscillations requires the inclusion of higher order terms in the analytical treatment of $\mathcal{A}$, which is beyond the scope of the present study.

Additionally, we numerically evaluate the terminal amplitude $\mathcal{A}\left[\Lambda^{n}, j_{y}\right]$, for various charge currents $j_{y}$ and dissipative torques $\Lambda^{n}$. The phase diagram [Fig. 3(a)] shows three regions: static, self-oscillations, and $2 \pi$ self-oscillations. The first two are separated by the threshold current $j_{\text {so }}^{(1)}$ shown in Fig. 3(b) for different values of damping. When $\left|\Lambda^{n}\right| / \Lambda$ is sufficiently large, the Néel field dynamics either vanish or proliferate into complete $2 \pi$ precessions. As the dissipative strength decreases, an intermediate window of stable (amplitude $<2 \pi$ ) oscillations open up: The lesser $\left|\Lambda^{n}\right|$ is, the wider the window is. In this region, the amplitude grows with increasing dissipative SOT, i.e., increasing $\left|\Lambda^{n}\right|$ and/or current density $j_{y}$. Eventually, when $j_{y}$ is sufficiently large, the oscillator enters the proliferation phase. The threshold current $j_{\text {so }}^{(2)}$ separates $\mathcal{A}<2 \pi$ from $\mathcal{A}=2 \pi$. If this threshold satisfies $j_{\text {so }}^{(2)}<4 j_{\text {sw }}$, the terminal amplitude has two possible outcomes $\mathcal{A}=0$ or $\mathcal{A}=2 \pi$. This region in the phase diagram is delimited by a red dashed line in Fig. 3(a). The color map therein represents the probability [33] to find: static solutions (red region) for currents close to $j_{\mathrm{so}}^{(2)}$ and $2 \pi$-amplitude oscillations (blue region) when the current is near $4 j_{\mathrm{sw}}$. This indeterminate behavior is due to local minima in $V[\phi]$ appearing at $\phi_{\mathrm{ss}}^{+}=-\pi / 2$ when $j_{\mathrm{sw}}<j_{y}<4 j_{\mathrm{sw}}$, see inset in Fig. 2(c). When the amplitude of self-oscillations reaches its maximum value, small increases on the current or small perturbations in the initial conditions can make the evolution of $\phi(t)$ to decay into $\phi_{\mathrm{ss}}^{+}$or proliferate in complete oscillations.

In the region $j_{\text {so }}^{(1)}<j_{y}<j_{\text {so }}^{(2)}$, the oscillator frequency has a nontrivial dependence on the current, as shown in Fig. 3(c). In contrast to the $2 \pi$-amplitude oscillating phase, the frequency of the $\mathcal{A}<2 \pi$ phase drops to a finite (nonzero) value at $j_{\text {so }}^{(2)}$. Also, a second, deeper drop occurs close to $j_{\text {so }}^{(1)}$ and it is deeper as the ratio $\left|\Lambda^{n}\right| / \Lambda$ decreases. When full oscillations occur, marked by $j_{y} \geqslant j_{\text {so }}^{(2)}$, the frequency monotonically grows and eventually becomes linear for large currents.

In the self-oscillating phase, a dc current controls the ac output power. This is because the Néel vector precesses around the direction of the applied dc current, either along the $x$ or the $y$ direction. Through the anisotropic magnetoresistance (AMR) [16,34], the relative change of the Néel vector with respect to the direction of current changes the induced voltage across the device. This transforms the self-oscillations into an oscillating voltage signal.

We predict that the self-oscillating phase appears for currents in the order of critical currents required for switching the magnetic moment. Measurements of the reactive and antidamping SOTs, predicted using symmetry arguments, will help to pave the way for self-oscillations in AFs. Recent measurements on the compensated ferrimagnet $\mathrm{Mn}_{2} \mathrm{Ru}_{x} \mathrm{Ga}$ [25-27] indicate a considerable dissipative SOT that enables the realization of our proposal. While current-induced magnetization dynamics studies in $\mathrm{Mn}_{2} \mathrm{Ru}_{x} \mathrm{Ga}$ are unexplored, we expect this material to exhibit a self-oscillating regime in the terahertz gap due to its low Gilbert damping, high anisotropy, and strong spin-orbit coupling. An additional advantage of this material is the possibility to tune the sign of the $\Lambda^{n} / \Lambda$ ratio by the compensation temperature as well as the prospect to use the stronger magnetoresistive effects associated with highly spin polarized materials to convert the dc charge current into an oscillating ac voltage.

\section{CONCLUSION AND DISCUSSION}

In conclusion, we have demonstrated that electrical currents can control antiferromagnetic self-oscillations. Using symmetry arguments on tetragonal crystalline antiferromagnets, we establish that SOTs contain reactive contributions and essential dissipative terms. By expressing the SOTs in terms of phenomenological parameters, we demonstrate that a nonlinear driven damped oscillator describes the antiferromagnetic dynamics. The dissipative SOT controls the effective damping enabling oscillations. When the charge current exceeds a threshold, self-oscillations spontaneously develop. An important feature of the self-oscillating phase is that the current density controls the ac output power. The Néel vector precesses around the direction of current. In turn, this 
generates an oscillating voltage signal via the anisotropic magnetoresistance effect (AMR) [16].

Note added. Recently, we became aware of another paper [35] that studied auto-oscillations in single permalloy layers using spin-orbit torques.

\section{ACKNOWLEDGMENTS}

R.T. would like to thank Alejandro Leon for discussions. This work was supported by the European Union's Horizon 2020 Research and Innovation Programme under Grant No. DLV-737038 "TRANSPIRE," and the Research Council of Norway through its Centres of Excellence funding scheme, Project No. 262633, "QuSpin," and the European Research Council via Advanced Grant No. 669442 "Insulatronics."

\section{APPENDIX A: PHENOMENOLOGY OF SPIN-ORBIT TORQUES}

In this section we compute current-induced SOTs for three examples of noncentrosymmetric tetragonal materials. The first one is the fully-compensated half-metallic ferrimagnet $\mathrm{Mn}_{2} \mathrm{Ru}_{x} \mathrm{Ga}$ (MRG) [25], while the second example belongs to the enantiomorphic crystals. Finally, we consider the antiferromagnetic materials CuMnAs [15] and $\mathrm{Mn}_{2} \mathrm{Au}$ [16]. We emphasize that the three classes of materials can be treated in the same framework by a simple rotation of current direction as is described below.

In linear response, the effective field induced by the outof-equilibrium current density $\mathbf{j}$ that is dependent on the local magnetization direction is $\mathbf{H}_{\alpha}=\boldsymbol{\eta}_{\alpha} \mathbf{j}$, where $\boldsymbol{\eta}$ is a tensor and $\alpha$ labels the sublattices. Thus, the torques at each sublattice are

$$
\begin{aligned}
\boldsymbol{\tau}_{a} & =\boldsymbol{m}_{a} \times \eta_{a} \mathbf{j}, \\
\boldsymbol{\tau}_{b} & =\boldsymbol{m}_{b} \times \boldsymbol{\eta}_{b} \mathbf{j} .
\end{aligned}
$$

Denoting $\mathcal{R}$ a symmetry rotation, the magnetization and current density obey $\boldsymbol{m}_{\alpha}^{\prime}=|\mathcal{R}| \mathcal{R} \boldsymbol{m}_{\alpha}$ and $\mathbf{j}^{\prime}=\mathcal{R} \mathbf{j}$, respectively, in the transformed frame. Based on the Neumann's principle [22] we found that the tensor $\boldsymbol{\eta}_{\alpha}$ satisfies the symmetry relations

$$
\begin{aligned}
& \boldsymbol{\eta}_{a}\left[\boldsymbol{m}_{a}^{\prime}, \boldsymbol{m}_{b}^{\prime}\right]=|\mathcal{R}| \mathcal{R} \boldsymbol{\eta}_{a}\left[\boldsymbol{m}_{a}, \boldsymbol{m}_{b}\right] \mathcal{R}^{T}, \\
& \boldsymbol{\eta}_{b}\left[\boldsymbol{m}_{a}^{\prime}, \boldsymbol{m}_{b}^{\prime}\right]=|\mathcal{R}| \mathcal{R} \boldsymbol{\eta}_{b}\left[\boldsymbol{m}_{a}, \boldsymbol{m}_{b}\right] \mathcal{R}^{T} .
\end{aligned}
$$

Assuming a series expansion in terms of powers of $m_{i}$ as $\left[\boldsymbol{\eta}_{a}\right]_{i j}=\left[\boldsymbol{\Lambda}_{a}\right]_{i j}+\left[\boldsymbol{\Lambda}_{a}^{a}\right]_{i j k} m_{a, k}+\left[\boldsymbol{\Lambda}_{a}^{b}\right]_{i j k} m_{b, k}$ and $\left[\boldsymbol{\eta}_{b}\right]_{i j}=$ $\left[\boldsymbol{\Lambda}_{b}\right]_{i j}+\left[\boldsymbol{\Lambda}_{b}^{a}\right]_{i j k} m_{a, k}+\left[\boldsymbol{\Lambda}_{b}^{b}\right]_{i j k} m_{b, k}$, we end up in the set of equations

$$
\begin{gathered}
{\left[\boldsymbol{\Lambda}_{a, b}\right]_{i j}=-|\mathcal{R}| \mathcal{R}_{i i^{\prime}} \mathcal{R}_{j j^{\prime}}\left[\boldsymbol{\Lambda}_{b, a}\right]_{i^{\prime} j^{\prime}},} \\
{\left[\boldsymbol{\Lambda}_{a, b}^{a}\right]_{i j k}=-\mathcal{R}_{i i^{\prime}} \mathcal{R}_{j j^{\prime}} \mathcal{R}_{k k^{\prime}}\left[\boldsymbol{\Lambda}_{b, a}^{b}\right]_{i^{\prime} j^{\prime} k^{\prime}} .}
\end{gathered}
$$

We consider the magnetic sublattices form inversion partners within each unit cell. This assumption leads to the additional condition

$$
\boldsymbol{\eta}_{b}\left[\boldsymbol{m}_{b}, \boldsymbol{m}_{a}\right]=-\boldsymbol{\eta}_{a}\left[\boldsymbol{m}_{a}, \boldsymbol{m}_{b}\right] .
$$

In the following we apply this result for the aforementioned example, thus reducing the unknown phenomenological coefficients of $\left[\boldsymbol{\Lambda}_{a, b}\right]_{i j},\left[\boldsymbol{\Lambda}_{a, b}^{a}\right]_{i j k}$, and $\left[\boldsymbol{\Lambda}_{b, a}^{b}\right]_{i j k}$ to a simple form.

\section{1. $\mathrm{Mn}_{2} \mathrm{Ru}_{x} \mathrm{Ga}$}

Here, we compute the SOTs solving Eqs. (A5)-(A7). Let us consider the fully-compensated ferrimagnet $\mathrm{Mn}_{2} \mathrm{Ru}_{x} \mathrm{Ga}$ (MRG). The point group of symmetries that leaves the sublattice site invariant is $\overline{4} 2 m$ [36]. The generators of all symmetries are

$$
\sigma^{(2)}=\left(\begin{array}{ccc}
-1 & 0 & 0 \\
0 & 1 & 0 \\
0 & 0 & -1
\end{array}\right), \quad \sigma^{(8)}=\left(\begin{array}{ccc}
0 & -1 & 0 \\
1 & 0 & 0 \\
0 & 0 & -1
\end{array}\right) .
$$

Solving Eqs. (A5) and (A6) for $\mathcal{R}=\sigma^{(2)}$ and $\mathcal{R}=\sigma^{(8)}$ we get the tensors $\boldsymbol{\eta}_{a}$ and $\boldsymbol{\eta}_{b}$. Therefore,

$$
\begin{aligned}
\boldsymbol{\eta}_{a}= & \Lambda\left(\begin{array}{ccc}
1 & 0 & 0 \\
0 & -1 & 0 \\
0 & 0 & 0
\end{array}\right)+\left(\begin{array}{ccc}
0 & \Lambda_{z}^{n} m_{z}^{a} & \Lambda_{y}^{n} m_{y}^{a} \\
\Lambda_{z}^{n} m_{z}^{a} & 0 & \Lambda_{y}^{n} m_{x}^{a} \\
\Lambda_{x}^{n} m_{y}^{a} & \Lambda_{x}^{n} m_{x}^{a} & 0
\end{array}\right) \\
& +\left(\begin{array}{ccc}
0 & \Lambda_{z}^{m} m_{z}^{b} & \Lambda_{y}^{m} m_{y}^{b} \\
\Lambda_{z}^{m} m_{z}^{b} & 0 & \Lambda_{y}^{m} m_{x}^{b} \\
\Lambda_{x}^{m} m_{y}^{b} & \Lambda_{x}^{m} m_{x}^{b} & 0
\end{array}\right)
\end{aligned}
$$

and

$$
\begin{aligned}
\boldsymbol{\eta}_{b}= & \bar{\Lambda}\left(\begin{array}{ccc}
1 & 0 & 0 \\
0 & -1 & 0 \\
0 & 0 & 0
\end{array}\right)+\left(\begin{array}{ccc}
0 & \bar{\Lambda}_{z}^{n} m_{z}^{a} & \bar{\Lambda}_{y}^{n} m_{y}^{a} \\
\bar{\Lambda}_{z}^{n} m_{z}^{a} & 0 & \bar{\Lambda}_{y}^{n} m_{x}^{a} \\
\bar{\Lambda}_{x}^{n} m_{y}^{a} & \bar{\Lambda}_{x}^{n} m_{x}^{a} & 0
\end{array}\right) \\
& +\left(\begin{array}{ccc}
0 & \bar{\Lambda}_{z}^{m} m_{z}^{b} & \bar{\Lambda}_{y}^{m} m_{y}^{b} \\
\bar{\Lambda}_{z}^{m} m_{z}^{b} & 0 & \bar{\Lambda}_{y}^{m} m_{x}^{b} \\
\bar{\Lambda}_{x}^{m} m_{y}^{b} & \bar{\Lambda}_{x}^{m} m_{x}^{b} & 0
\end{array}\right)
\end{aligned}
$$

are obtained.

The previous result can be substantially reduced assuming that the magnetic sublattices $\boldsymbol{m}_{a}$ and $\boldsymbol{m}_{b}$ form spin partners and that MRG is near the cubic phase. Although the former assumption is not valid for MRG, we consider it as an approximation in order to get the simplest expression for the torques. Finally, using the condition given by Eq. (A7) the torques acting on each sublattice are simply written as

$$
\begin{gathered}
\boldsymbol{\tau}_{a}=\Lambda \boldsymbol{m}_{a} \times \boldsymbol{j}+\mathcal{T}\left\{\boldsymbol{m}_{a} \times\left[\left(\Lambda^{n} \boldsymbol{m}_{a}+\Lambda^{m} \boldsymbol{m}_{b}\right) \times \boldsymbol{j}\right]\right\}, \\
\boldsymbol{\tau}_{b}=-\Lambda \boldsymbol{m}_{b} \times \boldsymbol{j}-\mathcal{T}\left\{\boldsymbol{m}_{b} \times\left[\left(\Lambda^{m} \boldsymbol{m}_{a}+\Lambda^{n} \boldsymbol{m}_{b}\right) \times \boldsymbol{j}\right]\right\},
\end{gathered}
$$

for an in-plane charge current $\boldsymbol{j}=\left(j_{x},-j_{y}, 0\right)$ and the operator $\mathcal{T}$ switches the sign of the $z$ component, while $x$ and $y$ components are unchanged.

\section{SOTs in enantiomorphic crystals}

Let us consider the point group of crystallographic symmetries as $422[13,23]$. The generators of symmetries are $\sigma^{(2)}$ and 
$\sigma^{(7)}$. The matricial forms of those transformations are [22]

$$
\sigma^{(2)}=\left(\begin{array}{ccc}
-1 & 0 & 0 \\
0 & 1 & 0 \\
0 & 0 & -1
\end{array}\right), \quad \sigma^{(7)}=\left(\begin{array}{ccc}
0 & 1 & 0 \\
-1 & 0 & 0 \\
0 & 0 & 1
\end{array}\right) \text {. }
$$

Solving Eqs. (A5)-(A7) for $\mathcal{R}=\sigma^{(2)}$ and $\mathcal{R}=\sigma^{(7)}$ we therefore get for the tensors $\boldsymbol{\eta}_{a}$ and $\boldsymbol{\eta}_{b}$,

$$
\begin{aligned}
\boldsymbol{\eta}_{a}= & \left(\begin{array}{ccc}
\Lambda & 0 & 0 \\
0 & \Lambda & 0 \\
0 & 0 & \Lambda_{z z}
\end{array}\right)+\left(\begin{array}{ccc}
0 & -\Lambda_{z}^{n} m_{z}^{a} & \Lambda_{y}^{n} m_{y}^{a} \\
\Lambda_{z}^{n} m_{z}^{a} & 0 & -\Lambda_{y}^{n} m_{x}^{a} \\
-\Lambda_{x}^{n} m_{y}^{a} & \Lambda_{x}^{n} m_{x}^{a} & 0
\end{array}\right) \\
& +\left(\begin{array}{ccc}
0 & -\Lambda_{z}^{m} m_{z}^{b} & \Lambda_{y}^{m} m_{y}^{b} \\
\Lambda_{z}^{m} m_{z}^{b} & 0 & -\Lambda_{y}^{m} m_{x}^{b} \\
-\Lambda_{x}^{m} m_{y}^{b} & \Lambda_{x}^{m} m_{x}^{b} & 0
\end{array}\right),
\end{aligned}
$$

and

$$
\begin{aligned}
\boldsymbol{\eta}_{b}= & \left(\begin{array}{ccc}
-\Lambda & 0 & 0 \\
0 & -\Lambda & 0 \\
0 & 0 & -\Lambda_{z z}
\end{array}\right)+\left(\begin{array}{ccc}
0 & \Lambda_{z}^{m} m_{z}^{a} & -\Lambda_{y}^{m} m_{y}^{a} \\
-\Lambda_{z}^{m} m_{z}^{a} & 0 & \Lambda_{y}^{m} m_{x}^{a} \\
\Lambda_{x}^{m} m_{y}^{a} & -\Lambda_{x}^{m} m_{x}^{a} & 0
\end{array}\right) \\
& +\left(\begin{array}{ccc}
0 & \Lambda_{z}^{n} m_{z}^{b} & -\Lambda_{y}^{n} m_{y}^{b} \\
-\Lambda_{z}^{n} m_{z}^{b} & 0 & \Lambda_{y}^{n} m_{x}^{b} \\
\Lambda_{x}^{n} m_{y}^{b} & -\Lambda_{x}^{n} m_{x}^{b} & 0
\end{array}\right) .
\end{aligned}
$$

To simplify the previous expressions, we consider that the tetragonal structure is slightly distorted with respect to the cubic phase. Therefore,

$$
\begin{aligned}
\boldsymbol{\eta}_{a}= & \Lambda\left(\begin{array}{lll}
1 & 0 & 0 \\
0 & 1 & 0 \\
0 & 0 & 1
\end{array}\right)+\Lambda^{n}\left(\begin{array}{ccc}
0 & -m_{z}^{a} & m_{y}^{a} \\
m_{z}^{a} & 0 & -m_{x}^{a} \\
-m_{y}^{a} & m_{x}^{a} & 0
\end{array}\right) \\
& +\Lambda^{m}\left(\begin{array}{ccc}
0 & -m_{z}^{b} & m_{y}^{b} \\
m_{z}^{b} & 0 & -m_{x}^{b} \\
-m_{y}^{b} & m_{x}^{b} & 0
\end{array}\right)
\end{aligned}
$$

and

$$
\begin{aligned}
\boldsymbol{\eta}_{b}= & -\Lambda\left(\begin{array}{ccc}
1 & 0 & 0 \\
0 & 1 & 0 \\
0 & 0 & 1
\end{array}\right)+\Lambda^{m}\left(\begin{array}{ccc}
0 & m_{z}^{a} & -m_{y}^{a} \\
-m_{z}^{a} & 0 & m_{x}^{a} \\
m_{y}^{a} & -m_{x}^{a} & 0
\end{array}\right) \\
& +\Lambda^{n}\left(\begin{array}{ccc}
0 & m_{z}^{b} & -m_{y}^{b} \\
-m_{z}^{b} & 0 & m_{x}^{b} \\
m_{y}^{b} & -m_{x}^{b} & 0
\end{array}\right) .
\end{aligned}
$$

Next, if we assume an in-plane current $\mathbf{j}$ the SOT's can be simply written as

$$
\begin{gathered}
\boldsymbol{\tau}_{a}=\Lambda \boldsymbol{m}_{a} \times \mathbf{j}+\boldsymbol{m}_{a} \times\left[\left(\Lambda^{n} \boldsymbol{m}_{a}+\Lambda^{m} \boldsymbol{m}_{b}\right) \times \mathbf{j}\right], \\
\boldsymbol{\tau}_{b}=-\Lambda \boldsymbol{m}_{b} \times \mathbf{j}-\boldsymbol{m}_{b} \times\left[\left(\Lambda^{m} \boldsymbol{m}_{a}+\Lambda^{n} \boldsymbol{m}_{b}\right) \times \mathbf{j}\right] .
\end{gathered}
$$

\section{SOTs in CuMnAs and $\mathrm{Mn}_{2} \mathrm{Au}$}

The symmetries that leave the magnetic sites in CuMnAs and $\mathrm{Mn}_{2} \mathrm{Au}$ belong to the polar point group $4 \mathrm{~mm}[13,23]$. The generators of symmetries are $\sigma^{(4)}$ and $\sigma^{(7)}$, and the matricial forms of those transformations are [22]

$$
\sigma^{(4)}=\left(\begin{array}{ccc}
1 & 0 & 0 \\
0 & -1 & 0 \\
0 & 0 & 1
\end{array}\right), \quad \sigma^{(7)}=\left(\begin{array}{ccc}
0 & 1 & 0 \\
-1 & 0 & 0 \\
0 & 0 & 1
\end{array}\right) .
$$

Solving Eqs. (A5)-(A7) for $\mathcal{R}=\sigma^{(4)}$ and $\mathcal{R}=\sigma^{(7)}$ we therefore get for the tensors $\boldsymbol{\eta}_{a}$ and $\boldsymbol{\eta}_{b}$,

$$
\begin{aligned}
\boldsymbol{\eta}_{a}= & \Lambda\left(\begin{array}{ccc}
0 & -1 & 0 \\
1 & 0 & 0 \\
0 & 0 & 0
\end{array}\right)+\left(\begin{array}{ccc}
\Lambda_{4}^{a} m_{z}^{a} & 0 & \Lambda_{1}^{a} m_{x}^{a} \\
0 & \Lambda_{4}^{a} m_{z}^{a} & \Lambda_{1}^{a} m_{y}^{a} \\
\Lambda_{3}^{a} m_{x}^{a} & \Lambda_{3}^{a} m_{y}^{a} & \Lambda_{2}^{a} m_{z}^{a}
\end{array}\right) \\
& +\left(\begin{array}{ccc}
\Lambda_{4}^{b} m_{z}^{b} & 0 & \Lambda_{1}^{b} m_{x}^{b} \\
0 & \Lambda_{4}^{b} m_{z}^{b} & \Lambda_{1}^{b} m_{y}^{b} \\
\Lambda_{3}^{b} m_{x}^{b} & \Lambda_{3}^{b} m_{y}^{b} & \Lambda_{2}^{b} m_{z}^{b}
\end{array}\right),
\end{aligned}
$$

and

$$
\begin{aligned}
\boldsymbol{\eta}_{b}= & -\Lambda\left(\begin{array}{ccc}
0 & -1 & 0 \\
1 & 0 & 0 \\
0 & 0 & 0
\end{array}\right)-\left(\begin{array}{ccc}
\Lambda_{4}^{b} m_{z}^{a} & 0 & \Lambda_{1}^{b} m_{x}^{a} \\
0 & \Lambda_{4}^{b} m_{z}^{a} & \Lambda_{1}^{b} m_{y}^{a} \\
\Lambda_{3}^{b} m_{x}^{a} & \Lambda_{3}^{b} m_{y}^{a} & \Lambda_{2}^{b} m_{z}^{a}
\end{array}\right) \\
& -\left(\begin{array}{ccc}
\Lambda_{4}^{a} m_{z}^{b} & 0 & \Lambda_{1}^{a} m_{x}^{b} \\
0 & \Lambda_{4}^{a} m_{z}^{b} & \Lambda_{1}^{a} m_{y}^{b} \\
\Lambda_{3}^{a} m_{x}^{b} & \Lambda_{3}^{a} m_{y}^{b} & \Lambda_{2}^{a} m_{z}^{b}
\end{array}\right) .
\end{aligned}
$$

Next, if we assume an in-plane current $\mathbf{j}$ and redefining the coefficients as $\Lambda_{4}^{a, b}=-\Lambda^{n, m}$ and $\Lambda_{3}^{a, b}=\Lambda^{n, m}+\underline{\Lambda}^{n, m}$, the SOT's can be simply written as

$$
\begin{aligned}
\boldsymbol{\tau}_{a}= & \Lambda \boldsymbol{m}_{a} \times(\boldsymbol{z} \times \mathbf{j})+\boldsymbol{m}_{a} \times\left[\left(\Lambda^{n} \boldsymbol{m}_{a}+\Lambda^{m} \boldsymbol{m}_{b}\right) \times(\boldsymbol{z} \times \mathbf{j})\right] \\
& +\boldsymbol{m}_{a} \times\left[\left(\underline{\Lambda}^{n} \boldsymbol{m}_{a} \cdot \mathbf{j}+\underline{\Lambda}^{m} \boldsymbol{m}_{b} \cdot \mathbf{j}\right) z\right], \\
\boldsymbol{\tau}_{b}= & -\Lambda \boldsymbol{m}_{b} \times(\boldsymbol{z} \times \mathbf{j})-\boldsymbol{m}_{b} \times\left[\left(\Lambda^{m} \boldsymbol{m}_{a}+\Lambda^{n} \boldsymbol{m}_{b}\right) \times(\boldsymbol{z} \times \mathbf{j})\right] \\
& -\boldsymbol{m}_{b} \times\left[\left(\underline{\Lambda}^{m} \boldsymbol{m}_{a} \cdot \mathbf{j}+\underline{\Lambda}^{n} \boldsymbol{m}_{b} \cdot \mathbf{j}\right) z\right] .
\end{aligned}
$$

\section{APPENDIX B: MULTIPLE-SCALE ANALYSIS}

In this section we apply multiple time-scales analysis [32] on Eq. (8), in the main text, to describe self-oscillations. Solutions of Eq. (8) exhibit a two-timing evolution, the slower and faster dynamics being the amplitude and precession evolution of the azimuthal angle, respectively. Through this framework, also extendable to multiple time scales [32], we can get rid of the faster time scale dynamics using perturbation theory. Thus, only the slow evolution of the azimuthal angle is captured by an amplitude and phase equation.

Let us consider the model equation of motion,

$$
\ddot{\phi}+\phi=\epsilon h(\phi, \dot{\phi})
$$

with $\epsilon$ a perturbative parameter and $h$ a nonlinear function of $\phi$ and $\dot{\phi}$. Next, consider the function $\phi$ characterized by having several time scales $\left\{T_{0}, T_{1}, \ldots, T_{N}\right\}$, where $N$ is determined by the modeling system. Those time scales are defined by the hierarchy: $T_{n} \equiv \epsilon^{n} t$ where $\epsilon$ is the perturbative parameter. Thus, the function $\phi\left(T_{0}, T_{1}, \ldots, T_{N}\right)$ and its total derivative 
obeys

$$
\begin{gathered}
\phi=\sum_{n=0}^{N} \epsilon^{n} \phi_{n}\left(T_{0}, T_{1}, \ldots, T_{N}\right) \\
\frac{d \phi}{d t}=\frac{\partial \phi}{\partial T_{0}}+\frac{\partial \phi}{\partial T_{1}} \epsilon+\cdots+\frac{\partial \phi}{\partial T_{N}} \epsilon^{N}=\sum_{n=0}^{N} \epsilon^{n} \frac{\partial \phi}{\partial T_{n}} .
\end{gathered}
$$

Replacing the two previous expressions for $\phi$ and $\dot{\phi}$ on Eq. (B1), we obtain

$$
\begin{aligned}
& \left(\sum_{m=0}^{N} \epsilon^{m} \frac{\partial}{\partial T_{m}}\right)^{2}\left(\sum_{n=0}^{N} \epsilon^{n} \phi_{n}\right)+\sum_{n=0}^{N} \epsilon^{n} \phi_{n} \\
& =\epsilon h\left(\sum_{n=0}^{N} \epsilon^{n} \phi_{n}, \sum_{m=0}^{N} \epsilon^{m} \frac{\partial}{\partial T_{m}}\left(\sum_{n=0}^{N} \epsilon^{n} \phi_{n}\right)\right),
\end{aligned}
$$

which is expanded at different orders in $\epsilon$, to get

$$
\begin{gathered}
\mathcal{O}\left(\epsilon^{0}\right) \Rightarrow\left(\frac{\partial^{2}}{\partial T_{0}^{2}}+1\right) \phi_{0}=0, \\
\mathcal{O}\left(\epsilon^{1}\right) \Rightarrow\left(\frac{\partial^{2}}{\partial T_{0}^{2}}+1\right) \phi_{1}=-2 \frac{\partial^{2} \phi_{0}}{\partial T_{0} \partial T_{1}}+h\left(\phi_{0}, \frac{\partial \phi_{0}}{\partial T_{0}}\right),
\end{gathered}
$$

and so on. To order $\epsilon^{0}$, we find the solution $\phi_{0}=$ $A \cos \left(T_{0}+\varphi\right)$, where the amplitude $A=A\left(T_{1}, \ldots T_{N}\right)$ and phase $\varphi=\varphi\left(T_{1}, \ldots T_{N}\right)$ are constants on time $T_{0}$ (but not necessarily on the rest of the temporal scales). Setting $\theta=T_{0}+\varphi$, we have for the equation to order $\epsilon^{1}$,

$$
\begin{aligned}
\left(\frac{\partial^{2}}{\partial \theta^{2}}+1\right) \phi_{1}= & 2 \frac{\partial A}{\partial T_{1}} \sin \theta+2 A \frac{\partial \varphi}{\partial T_{1}} \cos \theta \\
& +h(A \cos \theta,-A \sin \theta) .
\end{aligned}
$$

Although $h$ is unknown, it is periodic on $\theta$, and thus we may expand it in the Fourier series

$$
h(A \cos \theta,-A \sin \theta)=\sum_{k=1}^{\infty} \alpha_{k}(A) \sin k \theta+\sum_{k=0}^{\infty} \beta_{k}(A) \cos k \theta,
$$

with the Fourier coefficients $\alpha_{k}(A)$ and $\beta_{k}(A)$ to be determined. We now demand that secular terms, those proportional to $\sin \theta$ and $\cos \theta$ in Eq. (B6), must vanish. Therefore, the above implies

$$
2 \frac{\partial A}{\partial T_{1}}+\alpha_{1}(A)=0
$$

$$
2 A \frac{\partial \varphi}{\partial T_{1}}+\beta_{1}(A)=0 .
$$

The previous equations constitute a central result of this section. Through them, the temporal evolution of both amplitude and frequency are obtained. Next, we focus this analysis considering the nonlinear oscillator given by Eq. (8) in the main text. We consider the charge current along the $y$ direction and thus, the azimuthal angle varies around the steady state $\phi_{\mathrm{ss}}^{-}=\pi / 2$. For the sake of simplicity, we approximate the effective potential around $\phi_{\mathrm{ss}}^{-}$to a quadratic one. Therefore, the equation of motion reads in the form,

$$
\frac{d^{2} \phi}{d t^{2}}-\epsilon[1+\eta(1-\cos \phi)] \frac{d \phi}{d t}+\omega_{0}^{2} \phi=0,
$$

where the parameters are $\epsilon=\Lambda^{n} j_{y} / 2 \omega_{\mathrm{ex}}-\alpha, \omega_{0}^{2}=\Lambda j_{y}+$ $4 \omega_{\perp}^{(1)}$ and $\eta=\Lambda^{n} j_{y} /\left(\Lambda^{n} j_{y}-2 \omega_{\mathrm{ex}} \alpha\right)$. Here, the azimuthal angle depends on two time scales, $\phi=\phi\left(T_{f}, T_{s}\right)$, where $T_{f}$ and $T_{s}$ are the fast and slow time scales, respectively. We denote $\phi_{0}=\mathcal{A} \sin \theta$, with $\theta=T_{f}+\varphi$, the solution obtained up to $\epsilon^{0}$ order. Comparing Eqs. (B1) and (B10), we identify the nonlinear function as given by $h\left(\phi_{0}, \frac{\partial \phi_{0}}{\partial T_{f}}\right)=$ $-\omega_{0} \mathcal{A} \sin \theta\left[1-\eta\left(1-\cos \phi_{0}\right)\right]$. This function is expanded in Fourier series as

$$
\begin{aligned}
h\left(\phi_{0}, \frac{\partial \phi_{0}}{\partial T_{f}}\right)= & -\omega_{0}\left[\mathcal{A}+\eta \sum_{n=1}^{\infty}(-1)^{n} \frac{\mathcal{A}^{2 n+1}}{(2 n) !} \alpha_{1}^{n}\right] \sin \theta \\
& -\omega_{0} \eta \sum_{n=1}^{\infty} \sum_{k>1}(-1)^{n} \frac{\mathcal{A}^{2 n+1}}{(2 n) !} \alpha_{k}^{n} \sin k \theta .
\end{aligned}
$$

From Eqs. (B8) and (B9) we obtain for the amplitude and phase equations to obey

$$
\begin{gathered}
2 \frac{\partial \mathcal{A}}{\partial T_{s}}=\mathcal{A}+\eta \sum_{n=1}^{\infty}(-1)^{n} \frac{\mathcal{A}^{2 n+1}}{(2 n) !} \alpha_{1}^{n}, \\
\mathcal{A} \frac{\partial \varphi}{\partial T_{s}}=0,
\end{gathered}
$$

where the Fourier coefficient $\alpha_{1}^{n}$ is given by

$$
\alpha_{1}^{n}=2\left[\prod_{m=1}^{n} \frac{2(n-m)+1}{2(n-m)+2}-\prod_{m=1}^{n+1} \frac{2(n+1-m)+1}{2(n+1-m)+2}\right] .
$$

Up to leading order in the amplitude of oscillations, we get the well known Ginzburg-Landau equation with real coefficients

$$
2 \frac{\partial \mathcal{A}}{\partial T_{s}}=\mathcal{A}-\frac{\eta}{8} \mathcal{A}^{3}
$$

In the stationary limit we find the nonzero solution $\mathcal{A}=$ $2 \sqrt{2 / \eta}$. Defining $j_{\mathrm{so}}^{(1)}=2 \omega_{\mathrm{ex}} \alpha / \Lambda^{n}$, we can rewrite the steadystate solution as $\mathcal{A}=2 \sqrt{2} \sqrt{1-j_{\text {so }}^{(1)} / j_{y}}$.
[1] S. S. Dhillon, M. S. Vitiello, E. H. Linfield, A. G. Davies, M. C. Hoffmann, J. Booske, C. Paoloni, M. Gensch, P. Weightman, G. P. Williams, E. Castro-Camus, D. R. S.
Cumming, F. Simoens, I. Escorcia-Carranza, J. Grant, S. Lucyszyn, M. Kuwata-Gonokami, K. Konishi, M. Koch, C. A. Schmuttenmaer, T. L. Cocker, R. Huber, A. G. Markelz, Z. D. 
Taylor, V. P. Wallace, J. A. Zeitler, J. Sibik, T. M. Korter, B. Ellison, S. Rea, P. Goldsmith, K. B. Cooper, R. Appleby, D. Pardo, P. G. Huggard, V. Krozer, H. Shams, M. Fice, C. Renaud, A. Seeds, A. Stöhr, M. Naftaly, N. Ridler, R. Clarke, J. E. Cunningham, and M. B. Johnston, J. Phys. D 50, 043001 (2017).

[2] M. Tonouchi, Nat. Photonics 1, 97 (2007).

[3] A. S. Núñez, R. A. Duine, P. Haney, and A. H. MacDonald, Phys. Rev. B 73, 214426 (2006).

[4] V. Baltz, A. Manchon, M. Tsoi, T. Moriyama, T. Ono, and Y. Tserkovnyak, Rev. Mod. Phys. 90, 015005 (2018).

[5] T. Jungwirth, X. Marti, P. Wadley, and J. Wunderlich, Nat. Nanotechnol. 11, 231 (2016).

[6] S. A. Wolf, D. D. Awschalom, R. A. Buhrman, J. M. Daughton, S. von Molnár, M. L. Roukes, A. Y. Chtchelkanova, and D. M. Treger, Science 294, 1488 (2001).

[7] B. A. Ivanov, Low Temp. Phys. 40, 91 (2014).

[8] T. Satoh, S.-J. Cho, R. Iida, T. Shimura, K. Kuroda, H. Ueda, Y. Ueda, B. A. Ivanov, F. Nori, and M. Fiebig, Phys. Rev. Lett. 105, 077402 (2010).

[9] S. Wienholdt, D. Hinzke, and U. Nowak, Phys. Rev. Lett. 108, 247207 (2012).

[10] R. Cheng, M. W. Daniels, J.-G. Zhu, and D. Xiao, Phys. Rev. B 91, 064423 (2015).

[11] K. M. D. Hals and A. Brataas, Phys. Rev. B 88, 085423 (2013).

[12] J. Železný, H. Gao, K. Výborný, J. Zemen, J. Mašek, A. Manchon, J. Wunderlich, J. Sinova, and T. Jungwirth, Phys. Rev. Lett. 113, 157201 (2014).

[13] J. Železný, H. Gao, A. Manchon, F. Freimuth, Y. Mokrousov, J. Zemen, J. Mašek, J. Sinova, and T. Jungwirth, Phys. Rev. B 95, 014403 (2017).

[14] A. Manchon, I. Miron, T. Jungwirth, J. Sinova, J. Zelezny, A. Thiaville, K. Garello, and P. Gambardella, arXiv:1801.09636.

[15] P. Wadley, B. Howells, J. Železný, C. Andrews, V. Hills, R. P. Campion, V. Novák, K. Olejník, F. Maccherozzi, S. S. Dhesi, S. Y. Martin, T. Wagner, J. Wunderlich, F. Freimuth, Y. Mokrousov, J. Kuneš, J. S. Chauhan, M. J. Grzybowski, A. W. Rushforth, K. W. Edmonds, B. L. Gallagher, and T. Jungwirth, Science 351, 587 (2016).

[16] S. Y. Bodnar, L. Smejkal, I. Turek, T. Jungwirth, O. Gomonay, J. Sinova, A. A. Sapozhnik, H. J. Elmers, M. Klaui, and M. Jourdan, Nat. Commun. 9, 348 (2018).

[17] A. Jenkins, Phys. Rep. 525, 167 (2013).

[18] R. Cheng, D. Xiao, and A. Brataas, Phys. Rev. Lett. 116, 207603 (2016).

[19] R. Khymyn, I. Lisenkov, V. Tiberkevich, B. Ivanov, and A. A. Slavin, Sci. Rep. 7, 43705 (2017).
[20] O. R. Sulymenko, O. V. Prokopenko, V. S. Tiberkevich, A. N. Slavin, B. A. Ivanov, and R. S. Khymyn, Phys. Rev. Applied 8, 064007 (2017).

[21] J. Checiński, M. Frankowski, and T. Stobiecki, Phys. Rev. B 96, 174438 (2017).

[22] R. R. Birss, Symmetry and Magnetism (North-Holland, Amsterdam, 1966).

[23] T. Hahn (ed.), International Tables for Crystallography, Vol.A: Space Group Symmetry (Springer, New York, 2002).

[24] I. A. Ado, O. A. Tretiakov, and M. Titov, Phys. Rev. B 95, 094401 (2017).

[25] H. Kurt, K. Rode, P. Stamenov, M. Venkatesan, Y.-C. Lau, E. Fonda, and J. M. D. Coey, Phys. Rev. Lett. 112, 027201 (2014).

[26] K. Borisov, D. Betto, Y.-C. Lau, C. Fowley, A. Titova, N. Thiyagarajah, G. Atcheson, J. Lindner, A. M. Deac, J. M. D. Coey, P. Stamenov, and K. Rode, Appl. Phys. Lett. 108, 192407 (2016).

[27] M. Žic, K. Rode, N. Thiyagarajah, Y.-C. Lau, D. Betto, J. M. D. Coey, S. Sanvito, K. J. O'Shea, C. A. Ferguson, D. A. MacLaren, and T. Archer, Phys. Rev. B 93, 140202 (2016).

[28] For an in-plane current the dissipative torque on sublattice $a$ is expanded as $\boldsymbol{\tau}_{a}^{d}=\boldsymbol{m}_{a} \times\left[\Lambda^{\alpha} \boldsymbol{m}_{\alpha} \times(\boldsymbol{z} \times \mathbf{j})+\underline{\Lambda}^{\alpha}\left(\boldsymbol{m}_{\alpha} \cdot \mathbf{j}\right) z\right]$ As is shown in Refs. [12,13], when the Fermi level is close to the band gap $\Lambda^{\alpha} / \underline{\Lambda}^{\alpha} \gg 1$. Similar argument holds for the torque $\boldsymbol{\tau}_{b}^{d}$.

[29] $\mathbf{X}[\boldsymbol{n}, \dot{\boldsymbol{n}}]=-\left(\Lambda^{n} / 2 \omega_{\mathrm{ex}}\right)\left[2 \dot{n}_{z}(\boldsymbol{n} \cdot \boldsymbol{j}) \hat{z}+(\dot{\boldsymbol{n}} \cdot \boldsymbol{j}) \mathcal{T}\{\boldsymbol{n}\}\right]$. In the limit of large easy-plane anisotropy, $n_{z}$ is considered small. Therefore, the first term in the product $\boldsymbol{n} \times \mathbf{X}[\boldsymbol{n}, \dot{\boldsymbol{n}}]$ is negligible. We also disregard the second term, $\boldsymbol{n} \times \mathcal{T}\{\boldsymbol{n}\}$, since these vectors are approximately parallel.

[30] L. Perko, Differential Equations and Dynamical Systems, Third ed. (Springer-Verlag New York, 2001).

[31] Self-oscillations around $\phi_{\mathrm{ss}}^{+}$are obtained when $\Lambda^{n}>0$. These oscillations are highly unstable and thus are not considered.

[32] S. H. Strogatz, Nonlinear Dynamics and Chaos: With Applications to Physics, Biology, Chemistry, and Engineering (Westview Press, Boulder, 2015).

[33] This was determined averaging over a large amount of initial conditions $\phi(t=0)=\phi_{\mathrm{ss}}^{-}+\delta$, with $\delta$ randomly chosen in the interval [-0.02, 0.02].

[34] I. Fina, X. Marti, D. Yi, J. Liu, J.and Chu, C. Rayan-Serrao, S. Suresha, A. Shick, J. Železný, T. Jungwirth, J. Fontcuberta, and R. Ramesh, Nat. Commun. 5, 4671 (2014).

[35] M. Haidar, A. A. Awad, M. Dvornik, R. Khymyn, A. Houshang, and J. Akerman, arXiv:1808.09330.

[36] S. Lenne, Y.-C. Lau, A. Jah, J. M. D. Coey, P. Stamenov, K. Rode, R. E. Troncoso, and A. Brataas (unpublished). 